\title{
CR-submanifolds of a nearly trans-hyperbolic Sasakian manifold with a quarter symmetric non-metric connection
}

\author{
Mobin Ahmad*, Janardan Prasad Ojha ${ }^{* *}$ and Mohd. Danish Siddiqui* \\ *(Department of Mathematics, Jazan University, KSA) \\ *** (Department of Mathematics, SRMGPC, Lucknow-227105, India)
}

\begin{abstract}
This paper deals with the study of CR-submanifolds of a nearly trans-hyperbolic Sasakian manifold with a quarter symmetric non-metric connection. We study parallel distribution relating to $\xi$-vertical CRsubmanifolds of a nearly trans-hyperbolic Sasakian manifold with quarter symmetric non-metric connection. Further, we obtain the parallel distributions on CR-submanifolds.
\end{abstract}

Keywords: CR-submanifolds, nearly trans-hyperbolic Sasakian manifold, quarter symmetric non-metric connection, parallel distribution.

\section{Introduction}

In 1978, Aurel Bejancu introduced the notion of $C R$-submanifold of Kaehler manifold [1, 2]. On the other hand $C R$-submanifold have been studied by kobayashi [3]. J. A. Oubina introduced a new class of almost contact metric manifold known as trans-Sasakian manifold [4]. Gherghe studied on harmonicity on nearly transSasaki geometry of $C R$-submanifold of manifold [5]. $C R$-submanifold of a trans-Sasakian manifold have been studied by Shahid [6]. Later Al-Solamy studied the $C R$-submanifold of a nearly trans-Sasakian manifold [7]. In 1976, Upadhyay and Dube have studied almost contact hyperbolic structure [8]. Bhatt and Dube studied on $C R$ submanifold of trans-hyperbolic Sasakian manifold [9]. Gill and Dube have also worked on $C R$-submanifold of trans-hyperbolic Sasakian manifold [10]. Kumar and Dube studied $C R$-submanifold of a nearly trans-hyperbolic Sasakian manifold [11]. In this paper we study $C R$-submanifold of a nearly trans-hyperbolic Sasakian manifold endowed with a quarter symmetric non-metric connection. Let $\nabla$ be a linear connection in an $n$-dimensional differentiable manifold $M$. The torsion tensor $T$ and curvature tensor $R$ of $\nabla$ are given respectively by $T(X, Y)=\nabla_{X} Y-\nabla_{Y} X-[X, Y]$

$$
R(X, Y) Z=\nabla_{X} \nabla_{Y} Z-\nabla_{Y} \nabla_{X} Z-\nabla_{[X, Y]} Z
$$

The connection $\nabla$ is symmetric if its torsion tensor $T$ vanishes, otherwise it is non-symmetric. The connection $\nabla$ is metric connection if there is a Riemannian metric $g$ in $M$ such that $\nabla g=0$, otherwise it is non-metric. It is well known that a linear connection is symmetric and metric if and only if it is the Levi-Civita connection. In [12] S. Golab introduced the idea of a quarter symmetric connection. A linear connection is said to be a quarter symmetric connection if its torsion tensor $T$ is of the form $T(X, Y)=\eta(Y) \phi X-\eta(X) \phi Y$.

Some properties of quarter symmetric non-metric connection was studied by several authors in ([13], [14], [15], [16]).

This paper is organized as follows: In section 2, we give a brief introduction of nearly trans-hyperbolic Sasakian manifold. In section 3, we have proved some basic lemmas on nearly trans-hyperbolic Sasakian manifold with a quarter symmetric non-metric connection. In section 4 , we have discussed parallel distributions.

\section{Preliminaries}

Let $\bar{M}$ be an $n$-dimensional almost hyperbolic contact metric manifold with almost hyperbolic contact metric structure $(\phi, \xi, \eta, g)$, where a tensor $\phi$ of type $(1,1)$, a vector field $\xi$, called structure vector field and $\eta$, the dual 1-form of $\xi$ satisfying the following

for any $X, Y$ tangent to $\bar{M}$ [17]. In this case

$$
\begin{aligned}
& \phi^{2} X=X-\eta(X) \xi, \quad g(X, \xi)=\eta(X) \\
& \eta(\xi)=-1, \quad \phi(\xi)=0, \quad \eta \circ \phi=0 \\
& g(\phi X, \phi Y)=-g(X, Y)-\eta(X) \eta(Y)
\end{aligned}
$$




$$
g(\phi X, Y)=-g(X, \phi Y) .
$$

An almost hyperbolic contact metric structure $(\phi, \xi, \eta, g)$ on $\bar{M}$ is called trans-hyperbolic Sasakian [10] if and only if

$$
\left(\overline{\bar{\nabla}}_{X} \phi\right) Y=\alpha(g(X, Y) \xi-\eta(Y) \phi X)+\beta(g(\phi X, Y) \xi-\eta(Y) \phi X)
$$

for all $X, Y$ tangent to $\bar{M}$ and $\alpha, \beta$ are functions on $\bar{M}$. On a trans-hyperbolic Sasakian manifold $\bar{M}$, we have

$$
\overline{\bar{\nabla}}_{X} \xi=-\alpha(\phi X)+\beta(X-\eta(X) \xi),
$$

where $g$ is the Riemannian metric and $\overline{\bar{\nabla}}$ is the Riemannian connection.

Let $M$ be an $m$-dimensional isometrically immersed submanifold of nearly trans-hyperbolic Sasakian manifold $\bar{M}$. We denote by $g$ the Riemannian metric tensor field on $M$ as well as $\bar{M}$.

Definition 2.1. An $m$-dimensional Riemannian submanifold $M$ of a nearly trans-hyperbolic Sasakian manifold $\bar{M}$ is called a $C R$-submanifold if $\xi$ is tangent to $M$ and there exists differentiable distribution $D: x \in M \rightarrow D_{X} \subset T_{x}(M)$ such that

(i) the distribution $D_{x}$ is invariant under $\phi$, that is $\phi D_{x} \subset D_{x}$ for each $x \in M$;

(ii) the complementary orthogonal distribution $D^{\perp}: x \rightarrow D^{\perp}{ }_{x} \subset T_{x}(M)$ of the distribution $D$ on $M$ is anti-invariant under $\phi$ that is, $\phi D^{\perp}{ }_{x}(M) \subset T_{x}{ }^{\perp}(M)$ for all $x \in M$, where $T_{x}(M)$ and $T^{\perp}{ }_{x}(M)$ are tangent space and normal space of $M$ at $x \in M$ respectively.

If $\operatorname{dim} D^{\perp}{ }_{x}=0$ (resp. $\operatorname{dim} D_{x}=0$ ), then $C R$-submanifold is called an invariant (resp. anti-invariant). The distribution $D$ (resp. $D^{\perp}$ ) is called horizontal (resp. vertical) distribution. The pair $\left(D, D^{\perp}\right)$ is called $\xi$ horizontal (resp. $\xi$-vertical) if $\xi_{x} \in D_{x}$ (resp. $\xi_{x} \in D^{\perp}$ ) for $x \in M$.

For any vector field $X$ tangent to $M$, we write

$$
X=P X+Q X \text {, }
$$

where $P X$ and $Q X$ belong to the distribution $D$ and $D^{\perp}$ respectively.

For any vector field $N$ normal to $M$, we put

$$
\phi N=B N+C N,
$$

where $B N$ (resp. $C N$ ) denotes the tangential (resp. normal) component of $\phi N$.

Now, we remark that owing to the existence of the 1-form $\eta$, we can define a quarter symmetric non-metric connection $\bar{\nabla}$ in almost contact metric manifold by

$$
\bar{\nabla}_{X} Y=\overline{\bar{\nabla}}_{X} Y+\eta(Y) \phi X
$$

such that

$$
\left(\bar{\nabla}_{X} g\right)(Y, Z)=-\eta(Y) g(X, Z)-\eta(Z) g(X, Y)
$$

for any $X, Y \in T M$, where $\overline{\bar{\nabla}}$ is the induced connection with respect to $g$ on $M, \eta$ is a 1-form and $\xi$ is a vector field.

Using (2.5) and (2.10), we get

$$
\begin{aligned}
\left(\bar{\nabla}_{X} \phi\right) Y & =\alpha(g(X, Y) \xi-\eta(Y) \phi X)+\beta(g(\phi X, Y) \xi \\
& -\eta(Y) \phi X)-\eta(Y) X+\eta(Y) \eta(X) \xi .
\end{aligned}
$$

Similarly, we have

$$
\begin{aligned}
\left(\bar{\nabla}_{Y} \phi\right) X & =\alpha(g(Y, X) \xi-\eta(X) \phi Y)+\beta(g(\phi Y, X) \xi \\
& -\eta(X) \phi Y)-\eta(X) Y+\eta(X) \eta(Y) \xi .
\end{aligned}
$$

On adding above equations, we obtain 


$$
\begin{aligned}
\left(\bar{\nabla}_{X} \phi\right) Y+\left(\bar{\nabla}_{Y} \phi\right) X & =\alpha(2 g(X, Y) \xi-\eta(Y) \phi X-\eta(X) \phi Y) \\
& -\beta(\eta(X) \phi Y+\eta(Y) \phi X)-\eta(X) Y \\
& -\eta(Y) X+2 \eta(X) \eta(Y) \xi .
\end{aligned}
$$

This is the condition for an almost contact structure $(\phi, \xi, \eta, g)$ with a quarter symmetric non-metric connection to be nearly trans-hyperbolic Sasakian manifold.

From (2.10) and (2.6), we get

$$
\bar{\nabla}_{X} \xi=-(\alpha+1)(\phi X)+\beta(X-\eta(X) \xi) .
$$

We denote by $g$ the metric tensor of $\bar{M}$ as well as that induced on $M$. Let $\bar{\nabla}$ be the quarter symmetric non-metric connection on $\bar{M}$ and $\nabla$ be the induced connection on $M$ with respect to the unit normal $N$.

Theorem 2.2. The connection induced on the $C R$-submanifolds of a nearly trans-hyperbolic Sasakian manifold with a quarter symmetric non-metric connection is also a quarter symmetric non-metric connection.

Proof. Let $\nabla$ be the induced connection with respect to the unit normal $N$ on a $C R$-submanifold of a nearly trans-hyperbolic Sasakian manifold with a quarter symmetric non-metric connection $\bar{\nabla}$. Then

$$
\bar{\nabla}_{X} Y=\nabla_{X} Y+m(X, Y)
$$

where $m$ is a tensor field of type $(0,2)$ on $C R$-submanifold $M$. If $\nabla^{*}$ be the induced connection on $C R$ submanifolds from Riemannian connection $\overline{\bar{\nabla}}$, then

$$
\overline{\bar{\nabla}}_{X} Y=\nabla^{*}{ }_{X} Y+h(X, Y)
$$

where $h$ is a second fundamental tensor.

Now, from (2.14) and (2.15) we have

$$
\nabla_{X} Y+m(X, Y)=\nabla_{X}^{*} Y+h(X, Y)+\eta(Y) \phi X .
$$

Equating the tangential and normal components from both the sides in the above equation, we get

$$
h(X, Y)=m(X, Y)
$$

and

$$
\nabla_{X} Y=\nabla_{X}^{*} Y+\eta(Y) \phi X \text {. }
$$

Thus $\nabla$ is also a quarter symmetric non-metric connection.

Now, the Gauss formula for a $C R$-submanifold of a nearly trans-hyperbolic Sasakian manifold with a quarter symmetric non-metric connection is

$$
\bar{\nabla}_{X} Y=\nabla_{X} Y+h(X, Y)
$$

and the Weingarten formula for $M$ is given by

$$
\bar{\nabla}_{X} N=-A_{N} X+\nabla_{X}^{\perp} N
$$

for $X, Y \in T M, N \in T^{\perp} M$, where $h$ and $A$ are called the second fundamental tensors of $M$ and $\nabla^{\perp}$ denotes the operator of the normal connection. Moreover, we have

$$
g(h(X, Y), N)=g\left(A_{N} X, Y\right) .
$$

\section{Some Basic Lemmas}

Lemma 3.1 Let $M$ be a $C R$-submanifold of a nearly trans-hyperbolic Sasakian manifold $\bar{M}$ with a quartersymmetric non-metric connection. Then

$$
\begin{aligned}
& P\left(\nabla_{X} \phi P Y\right)+P\left(\nabla_{Y} \phi P X\right)-P\left(A_{\phi Q X} Y\right)-P\left(A_{\phi Q Y} X\right) \\
& =\phi P \nabla_{X} Y+\phi P \nabla_{Y} X+2 \alpha g(X, Y) P \xi-\alpha \eta(X) \phi P Y-\alpha \eta(Y) \phi P X \\
& -\beta \eta(Y) \phi P X-\beta \eta(X) \phi P Y-\eta(X) P Y-\eta(Y) P X+2 \eta(X) \eta(Y) P \xi, \\
& Q\left(\nabla_{X} \phi P Y\right)+Q\left(\nabla_{Y} \phi P X\right)-Q\left(A_{\phi Q X} Y\right)-Q\left(A_{\phi Q Y} X\right) \\
& =2 B h(X, Y)+2 \alpha g(X, Y) Q \xi-\eta(X) Q Y \\
& -\eta(Y) Q X+2 \eta(X) \eta(Y) Q \xi,
\end{aligned}
$$




$$
\begin{aligned}
& h(X, \phi P Y)+h(Y, \phi P X)+\nabla_{X}^{\perp} \phi Q Y+\nabla_{Y}^{\perp} \phi Q X \\
& =\phi Q \nabla_{Y} X+\phi Q \nabla_{X} Y+2 C h(X, Y)-(\alpha+\beta) \eta(Y) \phi Q X \\
& -(\alpha+\beta) \eta(X) \phi Q Y,
\end{aligned}
$$

for all $X, Y \in T M$.

Proof. By direct covariant differentiation, we have

$$
\begin{aligned}
& \bar{\nabla}_{X} \phi Y=\left(\bar{\nabla}_{X} \phi\right) Y+\phi \nabla_{X} Y+\phi h(X, Y), \\
& \bar{\nabla}_{X} \phi Y=\bar{\nabla}_{X} \phi P Y+\bar{\nabla}_{X} \phi Q Y .
\end{aligned}
$$

By virtue of (2.8), (2.11), (2.16) and (2.17), we get

$$
\begin{aligned}
\left(\bar{\nabla}_{X} \phi\right) Y+\phi \nabla_{X} Y+\phi h(X, Y) & =P \nabla_{X} \phi P Y+Q \nabla_{X} \phi P Y+h(X, \phi P Y) \\
& +\nabla_{X}^{\perp} \phi Q Y-P A_{\phi Q Y} X-Q A_{\phi Q Y} X . \\
\left(\bar{\nabla}_{Y} \phi\right) X+\phi \nabla_{Y} X+\phi h(Y, X) & =P \nabla_{Y} \phi P X+Q \nabla_{Y} \phi P X+h(Y, \phi P X) \\
& +\nabla_{Y}^{\perp} \phi Q X-P A_{\phi Q X} Y-Q A_{\phi Q X} Y .
\end{aligned}
$$

Similarly,

Adding, we obtain

$$
\begin{aligned}
& \left(\left(\bar{\nabla}_{X} \phi\right) Y+\left(\bar{\nabla}_{Y} \phi\right) X\right)+\phi P \nabla_{X} Y+\phi Q \nabla_{X} Y+\phi P \nabla_{Y} X+\phi Q \nabla_{Y} X \\
& +2 B h(X, Y)+2 C h(X, Y)=\alpha(2 g(X, Y) P \xi+\alpha(2 g(X, Y) Q \xi \\
& -\alpha \eta(Y) \phi P X-\alpha \eta(Y) \phi Q X-\alpha \eta(X) \phi P Y-\alpha \eta(X) \phi Q Y \\
& -\beta \eta(X) \phi P Y-\beta \eta(X) \phi Q Y-\beta \eta(Y) \phi Q X-\beta \eta(Y) \phi P X \\
& -\eta(X) P Y-\eta(X) Q Y-\eta(Y) P X-\eta(Y) Q X+2 \eta(X) \eta(Y) P \xi \\
& +2 \eta(X) \eta(Y) Q \xi+\phi P \nabla_{X} Y+\phi Q \nabla_{X} Y+\phi P \nabla_{Y} X+\phi Q \nabla_{Y} X \\
& +2 B h(X, Y)+2 C h(X, Y)
\end{aligned}
$$

for any $X, Y \in T M$.

Now, equating horizontal, vertical and normal components in (3.4) we get the desired result.

Lemma 3.2. Let $M$ be a $C R$-Submanifod of a nearly trans-hyperbolic Sasakian manifold $\bar{M}$ with a quarter symmetric non-metric connection. Then

$$
\begin{aligned}
2\left(\bar{\nabla}_{X} \phi\right) Y & \left.=\nabla_{X} \phi Y-\nabla_{Y} \phi X+h(X, \phi Y)\right)-h(Y, \phi X)-\phi[X, Y] \\
& +\alpha(2 g(X, Y) \xi-\eta(Y) \phi X+\eta(X) \phi Y)-\beta(\eta(X) \phi Y \\
& +\eta(Y) \phi X)-(\eta(Y) X+\eta(X) Y-2 \eta(X) \eta(Y) \xi), \\
2\left(\bar{\nabla}_{Y} \phi\right) X & =\alpha(2 g(X, Y) \xi-\eta(Y) \phi X-\eta(X) \phi Y)-\beta(\eta(X) \phi Y \\
& +\eta(Y) \phi X)-(\eta(X) Y+\eta(Y) X-2 \eta(X) \eta(Y) \xi)-\left(\nabla_{X} \phi Y\right) \\
& -h(X, \phi Y)+\nabla_{Y} \phi X+h(Y, \phi X)+\phi[X, Y]
\end{aligned}
$$

for any $X, Y \in D$.

Proof. From Gauss formula (2.16), we have

Also, we have

$$
\bar{\nabla}_{X} \phi Y-\bar{\nabla}_{Y} \phi X=\nabla_{X} \phi Y+h(X, \phi Y)-\nabla_{Y} \phi X-h(Y, \phi X) .
$$

From (3.7) and (3.8), we get

$$
\begin{aligned}
\left(\bar{\nabla}_{X} \phi\right) Y-\left(\bar{\nabla}_{Y} \phi\right) X & =\nabla_{X} \phi Y+h(X, \phi Y)-\nabla_{Y} \phi X \\
& -h(Y, \phi X)-\phi[X, Y] .
\end{aligned}
$$

Also for nearly trans-hyperbolic Sasakian manifold with quarter symmetric non-metric connection, we have 


$$
\begin{aligned}
\left(\bar{\nabla}_{X} \phi\right) Y+\left(\bar{\nabla}_{Y} \phi\right) X & =\alpha(2 g(X, Y) \xi-\eta(Y) \phi X-\eta(X) \phi Y) \\
& -\beta(\eta(X) \phi Y+\eta(Y) \phi X)-(\eta(Y) X \\
& +\eta(X) Y-2 \eta(X) \eta(Y) \xi .
\end{aligned}
$$

Adding (3.9) and (3.10), we obtain

$$
\begin{aligned}
2\left(\bar{\nabla}_{X} \phi\right) Y & \left.=\nabla_{X} \phi Y-\nabla_{Y} \phi X+h(X, \phi Y)\right)-h(Y, \phi X)-\phi[X, Y] \\
& +\alpha(2 g(X, Y) \xi-\eta(Y) \phi X+\eta(X) \phi Y)-\beta(\eta(X) \phi Y \\
& +\eta(Y) \phi X)-(\eta(Y) X+\eta(X) Y-2 \eta(X) \eta(Y) \xi) .
\end{aligned}
$$

Subtracting (3.9) from (3.10), we get

$$
\begin{aligned}
2\left(\bar{\nabla}_{Y} \phi\right) X & =\alpha(2 g(X, Y) \xi-\eta(Y) \phi X-\eta(X) \phi Y)-\beta(\eta(X) \phi Y \\
& +\eta(Y) \phi X)-(\eta(X) Y+\eta(Y) X-2 \eta(X) \eta(Y) \xi) \\
& -\left(\nabla_{X} \phi Y\right)-h(X, \phi Y)+\nabla_{Y} \phi X+h(Y, \phi X)+\phi[X, Y] .
\end{aligned}
$$

Hence Lemma is proved.

Lemma 3.3. Let $M$ be a $C R$-submanifold of a nearly trans-hyperbolic Sasakian manifold $\bar{M}$ with a quarter symmetric non-metric connection, then

$$
\begin{aligned}
2\left(\bar{\nabla}_{Y} \phi\right) Z & =A_{\phi Y} Z-A_{\phi Z} Y+\nabla_{Y}^{\perp} \phi Z-\nabla_{Z}^{\perp} \phi Y-\phi[Y, Z]+\alpha(2 g(Y, Z) \xi \\
& -\eta(Y) \phi Z-\eta(Z) \phi Y)-\beta(\eta(Y) \phi Z+\eta(Z) \phi Y)-(\eta(Y) Z \\
& +\eta(Z) Y-2 \eta(Y) \eta(Z) \xi)
\end{aligned}
$$

and

$$
\begin{aligned}
2\left(\bar{\nabla}_{Z} \phi\right) Y & =-A_{\phi Y} Z+A_{\phi Z} Y-\nabla_{Y}^{\perp} \phi Z+\nabla_{Z}^{\perp} \phi Y+\phi[Y, Z]+\alpha(2 g(Y, Z) \xi \\
& -\eta(Y) \phi Z-\eta(Z) \phi Y)-\beta(\eta(Y) \phi Z+\eta(Z) \phi Y)-(\eta(Y) Z \\
& +\eta(Z) Y-2 \eta(Y) \eta(Z) \xi)
\end{aligned}
$$

for any $Y, Z \in D^{\perp}$.

Proof. From Weingarten formula (2.17), we have

$$
\bar{\nabla}_{Z} \phi Y-\bar{\nabla}_{Y} \phi Z=A_{\phi Y} Z-A_{\phi Z} Y+\nabla_{Y}^{\perp} \phi Z-\nabla_{Z}^{\perp} \phi Y \text {. }
$$

Also, we have

$$
\bar{\nabla}_{Z} \phi Y-\bar{\nabla}_{Y} \phi Z=\left(\bar{\nabla}_{Y} \phi\right) Z-\left(\bar{\nabla}_{Z} \phi\right) Y+\phi[Y, Z] .
$$

From (3.11) and (3.12), we get

$$
\begin{aligned}
\left(\bar{\nabla}_{Y} \phi\right) Z+\left(\bar{\nabla}_{Z} \phi\right) Y & =\alpha(2 g(Y, Z) \xi-\eta(Y) \phi Z-\eta(Z) \phi Y) \\
& -\beta(\eta(Y) \phi Z+\eta(Z) \phi Y)-(\eta(Y) Z \\
& +\eta(Z) Y-2 \eta(Y) \eta(Z) \xi)
\end{aligned}
$$

On adding (3.13) and (3.14), we obtain

$$
\begin{aligned}
2\left(\bar{\nabla}_{Y} \phi\right) Z & =A_{\phi Y} Z-A_{\phi Z} Y+\nabla_{Y}^{\perp} \phi Z-\nabla_{Z}^{\perp} \phi Y-\phi[Y, Z] \\
& +\alpha(2 g(Y, Z) \xi-\eta(Y) \phi Z-\eta(Z) \phi Y)-\beta(\eta(Y) \phi Z \\
& +\eta(Z) \phi Y)-(\eta(Y) Z+\eta(Z) Y-2 \eta(Y) \eta(Z) \xi) .
\end{aligned}
$$

Subtracting (3.13) and (3.14), we find

$$
\begin{aligned}
2\left(\bar{\nabla}_{Z} \phi\right) Y & =-A_{\phi Y} Z+A_{\phi Z} Y-\nabla_{Y}^{\perp} \phi Z+\nabla_{Z}^{\perp} \phi Y+\phi[Y, Z] \\
& +\alpha(2 g(Y, Z) \xi-\eta(Y) \phi Z-\eta(Z) \phi Y)-\beta(\eta(Y) \phi Z \\
& +\eta(Z) \phi Y)-(\eta(Y) Z+\eta(Z) Y-2 \eta(Y) \eta(Z) \xi) .
\end{aligned}
$$

This proves our assertions.

Lemma 3.4. Let $M$ be a $C R$-submanifold of a nearly trans-hyperbolic Sasakian manifold $\bar{M}$ with a quarter symmetric non-metric connection, then 


$$
\begin{aligned}
2\left(\bar{\nabla}_{X} \phi\right) Y & =-A_{\phi Y} X+\nabla_{X}^{\perp} \phi Y-\nabla_{Y} \phi X-h(Y, \phi X)-\phi[X, Y] \\
& +\alpha(2 g(X, Y) \xi-\eta(Y) \phi X-\eta(X) \phi Y)-\beta(\eta(X) \phi Y \\
& +\eta(Y) \phi X)-(\eta(X) Y+\eta(Y) X-2 \eta(X) \eta(Y) \xi), \\
2\left(\bar{\nabla}_{Y} \phi\right) X & =A_{\phi Y} X-\nabla_{X}^{\perp} \phi Y+\nabla_{Y} \phi X+h(Y, \phi X)+\phi[X, Y]+\alpha(2 g(X, Y) \xi \\
& -\eta(Y) \phi X-\eta(X) \phi Y)-\beta(\eta(X) \phi Y+\eta(Y) \phi X)
\end{aligned}
$$

for any $X \in D$ and $Y \in D^{\perp}$.

Proof. By using Gauss and Weingarten equation for $X \in D$ and $Y \in D^{\perp}$ respectively, we get

$$
\bar{\nabla}_{X} \phi Y-\bar{\nabla}_{Y} \phi X=-A_{\phi Y} X+\nabla_{X}^{\perp} \phi Y-\nabla_{Y} \phi X-h(Y, \phi X) \text {. }
$$

Also, we have

$$
\bar{\nabla}_{X} \phi Y-\bar{\nabla}_{Y} \phi X=\left(\bar{\nabla}_{X} \phi\right) Y-\left(\bar{\nabla}_{Y} \phi\right) X+\phi[X, Y] .
$$

From (3.15) and (3.16), we obtain

$$
\left(\bar{\nabla}_{X} \phi\right) Y-\left(\bar{\nabla}_{Y} \phi\right) X=-A_{\phi Y} X+\nabla_{X}^{\perp} \phi Y-\nabla_{Y} \phi X-h(Y, \phi X)-\phi[X, Y] .
$$

Also for nearly trans-hyperbolic Sasakian manifold with a quarter symmetric non-metric connection, we have

$$
\begin{aligned}
\left(\bar{\nabla}_{X} \phi\right) Y+\left(\bar{\nabla}_{Y} \phi\right) X & =\alpha(2 g(X, Y) \xi-\eta(Y) \phi X-\eta(X) \phi Y)-\beta(\eta(X) \phi Y \\
& +\eta(Y) \phi X)-(\eta(X) Y+\eta(Y) X-2 \eta(X) \eta(Y) \xi) .
\end{aligned}
$$

Adding (3.17) and (3.18), we find

$$
\begin{aligned}
2\left(\bar{\nabla}_{X} \phi\right) Y & =-A_{\phi Y} X+\nabla_{X}^{\perp} \phi Y-\nabla_{Y} \phi X-h(Y, \phi X)-\phi[X, Y] \\
& +\alpha(2 g(X, Y) \xi-\eta(Y) \phi X-\eta(X) \phi Y)-\beta(\eta(X) \phi Y \\
& +\eta(Y) \phi X)-(\eta(X) Y+\eta(Y) X-2 \eta(X) \eta(Y) \xi) .
\end{aligned}
$$

Subtracting (3.17) from (3.18), we get

$$
\begin{aligned}
2\left(\bar{\nabla}_{Y} \phi\right) X & =A_{\phi Y} X-\nabla_{X}^{\perp} \phi Y+\nabla_{Y} \phi X+h(Y, \phi X)+\phi[X, Y]+\alpha(2 g(X, Y) \xi \\
& -\eta(Y) \phi X-\eta(X) \phi Y)-\beta(\eta(X) \phi Y+\eta(Y) \phi X) .
\end{aligned}
$$

Hence Lemma is proved.

\section{Parallel Distributions}

Definition 4.1. The horizontal (resp. vertical) distribution $D$ (resp. $D^{\perp}$ ) is said to be parallel with respect to the quarter symmetric non-metric connection on $M$ if $\nabla_{X} Y \in D$ (resp. $\nabla_{Z} W \in D^{\perp}$ ) for any vector field $X, Y \in D$ (resp. $W, Z \in D^{\perp}$ ).

Proposition 4.1. Let $M$ be a $\xi$-vertical $C R$-submanifold of a nearly trans-hyperbolic Sasakian manifold $\bar{M}$ with a quarter symmetric non-metric connection. If the horizontal distribution $D$ is parallel, then

$$
h(X, \phi Y)=h(Y, \phi X)
$$

for all $X, Y \in D$.

Proof. For horizontal distribution $D$, we have

$$
\nabla_{X} \phi Y \in D, \nabla_{Y} \phi X \in D \text { for any } X, Y \in D .
$$

Using the fact that $Q X=Q Y=0$ for $X, Y \in D$, (3.2) gives

$$
B h(X, Y)=-\alpha g(X, Y) Q \xi, \quad \text { for any } X, Y \in D \text {. }
$$

Also, since

Therefore,

From (3.3), we have

$$
\phi h(X, Y)=B h(X, Y)+C h(X, Y),
$$$$
\phi h(X, Y)=-\alpha g(X, Y) Q \xi+C h(X, Y) \text { for any } X, Y \in D \text {. }
$$

$$
h(X, \phi Y)+h(Y, \phi X)=2 C h(X, Y)=2 \phi h(X, Y)+2 \alpha g(X, Y) Q \xi
$$


for any $X, Y \in D$. Putting $X=\phi X \in D$ in (4.6), we get

$$
h(\phi X, \phi Y)+h\left(Y, \phi^{2} X\right)=2 \phi h(\phi X, Y)+2 \alpha g(\phi X, Y) Q \xi
$$

or

$$
h(\phi X, \phi Y)-h(Y, X)=2 \phi h(\phi X, Y)+2 \alpha g(\phi X, Y) Q \xi .
$$

Similarly, putting $Y=\phi Y \in D$ in (4.6), we find

$$
h(\phi Y, \phi X)-h(X, Y)=2 \phi h(X, \phi Y)+2 \alpha g(X, \phi Y) Q \xi .
$$

Hence from (4.8) and (4.9), we have

$$
\phi h(X, \phi Y)-\phi h(Y, \phi X)=\alpha g(\phi X, Y) Q \xi-\alpha g(X, \phi Y) Q \xi .
$$

Operating $\phi$ on both sides of (4.10) and using $\phi \xi=0$, we get

$$
h(X, \phi Y)=h(Y, \phi X)
$$

for all $X, Y \in D$.

Now, for the distribution $D^{\perp}$ we prove the following proposition.

Proposition 4.2. Let $M$ be a $\xi$-vertical $C R$-submanifold of a nearly trans-hyperbolic Sasakian manifold $\bar{M}$ with a quarter symmetric non-metric connection. If the distribution $D^{\perp}$ is parallel with respect to the connection on $M$, then

$$
A_{\phi Y} Z+A_{\phi Z} Y \in D^{\perp} \text { for any } Y, Z \in D^{\perp} .
$$

Proof. Using Gauss and Weingarten formula, we obtain

$$
\begin{aligned}
& -A_{\phi Z} Y+\nabla_{Y}^{\perp} \phi Z-A_{\phi Y} Z+\nabla_{Z}^{\perp} \phi Y=\phi \nabla_{Y} Z+\phi \nabla_{Z} Y+2 \phi h(Y, Z) \\
& +\alpha(2 g(Y, Z) \xi-\eta(Y) \phi Z-\eta(Z) \phi Y)-\beta(\eta(Z) \phi Y+\eta(Y) \phi Z) \\
& -(\eta(Y) Z+\eta(Z) Y-2 \eta(Y) \eta(Z) \xi)
\end{aligned}
$$

for any $Y, Z \in D^{\perp}$. Taking inner product with $X \in D$ in (3.13), we get

$$
g\left(A_{\phi Y} Z, X\right)+g\left(A_{\phi Z} Y, X\right)=g\left(\nabla_{Y} Z, \phi X\right)+g\left(\nabla_{Z} Y, \phi X\right) .
$$

If the distribution $D^{\perp}$ is parallel, then $\nabla_{Y} Z \in D^{\perp}$ and $\nabla_{Z} Y \in D^{\perp}$ for any $Y, Z \in D^{\perp}$.

So from (4.14), we get

$$
g\left(A_{\phi Y} Z, X\right)+g\left(A_{\phi Z} Y, X\right)=0 \text { or } g\left(A_{\phi Y} Z+A_{\phi Z} Y, X\right)=0
$$

which is equivalent to

$$
A_{\phi Y} Z+A_{\phi Z} Y \in D^{\perp}
$$

for any $Y, Z \in D^{\perp}$.

This completes the proof.

Definition 4.3. A $C R$-submanifold with a quarter-symmetric non-metric connection is said to be mixed totally geodesic if $h(X, Z)=0$ for all $X \in D$ and $Z \in D^{\perp}$.

The following Lemma is an easy consequence of (2.18).

Lemma 4.4. Let $M$ be a $C R$-submanifold of a nearly trans-hyperbolic Sasakian manifold $\bar{M}$ with a quartersymmetric non-metric connection. Then $M$ is mixed totally geodesic if and only if $A_{N} X \in D$ for all $X \in D$.

Definition 4.5. A normal vector field $N \neq 0$ is called $D$-parallel normal section if $\nabla_{X}^{\perp} N=0$ for all $X \in D$.

Now, we have the following proposition.

Proposition 4.6. Let $M$ be a mixed totally geodesic $\xi$-vertical $C R$-submanifold of a nearly trans-hyperbolic Sasakian manifold $\bar{M}$ with a quarter symmetric non-metric connection. Then the normal section $N \in \phi D^{\perp}$ is $D$-parallel if and only if $\nabla_{X} \phi N \in D$ for all $X \in D$.

Proof. Let $N \in \phi D^{\perp}$. Then from (3.2), we have 


$$
Q\left(\nabla_{Y} \phi X\right)=0 \text { for any } X \in D, Y \in D^{\perp} \text {. }
$$

In particular, we have

$$
Q\left(\nabla_{Y} X\right)=0 \text {. }
$$

By using it in (3.3), we get

$$
\nabla_{X}^{\perp} \phi Q Y=\phi Q \nabla_{X} Y \quad \text { or } \quad \nabla_{X}^{\perp} N=-\phi Q \nabla_{X} \phi N \text {. }
$$

Thus, if the normal section $N \neq 0$ with quarter symmetric non-metric connection is $D$-parallel, then by definition and (4.18), we get

$$
\phi Q\left(\nabla_{X} \phi N\right)=0
$$

which is equivalent to $\nabla_{X} \phi N \in D$ for all $X \in D$.

The converse part easily follows from (4.18).

This completes the proof of the proposition.

\section{References}

[1]. Bejancu, $C R$-submsnifolds of a Kaehler manifold. I, Proc. Amer. Math. Soc. 69 (1978), 135-142

[2]. A. Bejancu, Geometry of $C R$-submanifolds, D. Reidel Publishing Company, Holland, 1986.

[3]. M. Kobayashi, $C R$-submanifolds of Sasakian manifold, Tensor (N.S.) 35 (1981), no. 3, 297-307.

[4]. J. A. Oubina, New class of almost contact metric structures, Publ. Math. Debrecen, 32 (1985), 187-193.

[5]. C. Gherghe, Harmonicity on nearly trans-Sasaki manifolds, Demonstratio Math. 33(2000), no. 1, $151-157$.

[6]. M. H. Shahid, $C R$-submanifolds of a trans-Sasakian manifold, Indian J. Pure Appl. Math. 22 (1991), 1007-1012.

[7]. F. R. Al-Solamy, CR-submanifolds of a nearly trans-Sasakian manifold, IJMMS, 31 (3) (2002), 167-175.

[8]. M. D. Upadhyay and K. K. Dube, Almost contact hyperbolic $(f, g, \eta, \xi)$-structure, Acta. Math. Acad. Scient. Hung., Tomus 28 (1976), 1-4

[9]. L. Bhatt and K. K. Dube, On CR-submanifolds of a trans-hyperbolic Sasakian manifold, Acta Ciencia Indica 31 (2003), $91-96$.

[10]. H. S. Gill and K. K. Dube, On CR-submanifolds of trans-hyperbolic Sasakian manifolds, Demonstratio Math. 38 (2005), $953-960$.

[11]. S. Kumar and K. K. Dube, CR-submanifolds of a nearly trans-hyperbolic Sasakian manifold, Demonstratio Mathematica, Vol. XLI No .4 (2008), 922-929.

[12]. S. Golab, On semi symmetric and Quarter symmetric linear connections. Tensor 29 (1975), 249-254.

[13]. M. Ahmad, M. D. Siddiqui and J. P. Ojha, Semi-invariant submanifolds of Kenmotsu manifolds immersed in a generalized almost r-contact structure admitting a quarter symmetric non-metric connection, J. Math. comput. Sci. 2 (2012), No.4, 982-998.

[14]. M. Ahmad, CR-submanifolds of LP-Sasakian manifolds endowed with a quarter symmetric metric connection, Bull. Korean Math. Soc. 49 (2012), No.1, 25-32.

[15]. M. Ahmad, J. B. Jun and A. Haseeb, Submanifolds of an almost r-paracontact Reimannian manifold endowed with a quarter symmetric non-metric connection, J. Chungcheong Math. Soc. Vol. 24, No.1 (2011), 91-104.

[16]. M. Ahmad, C. Ozgur and A. Haseeb, Hypersurfaces of an almost r-paracontact Reimannian manifold endowed with a quarter symmetric non-metric connection. Kyungpook Math. j., 49 (2009), 533-543.

[17]. D. E. Blair, Contact manifolds in Riemannian Geometry, Lecture Notes in Mathematics, vol. 509, Springer-Verlag, Berlin, 1976. 